\title{
Solitary cardiac metastasis of uterine cervical cancer with antemortem diagnosis: A case report and literature review
}

\author{
KEISUKE TSUCHIDA ${ }^{1}$, TAKAHIRO OIKE ${ }^{1}$, TOSHIYUKI OHTSUKA ${ }^{2}$, \\ MUNENORI IDE $^{3}$, YOSUKE TAKAKUSAGI ${ }^{1}$, SHIN-EI NODA ${ }^{1}$, TOMOAKI TAMAKI ${ }^{1}$, \\ NOBUTERU KUBO $^{1}$, YUKA HIROTA ${ }^{1}$, TATSUYA OHNO ${ }^{1}$ and TAKASHI NAKANO ${ }^{1}$ \\ ${ }^{1}$ Department of Radiation Oncology, Gunma University Graduate School of Medicine, Maebashi, Gunma 371-8511; \\ ${ }^{2}$ Department of Internal Medicine, National Hospital Organization Numata National Hospital, Numata, Gunma 378-0051; \\ ${ }^{3}$ Department of Pathology, Maebashi Red Cross Hospital, Maebashi, Gunma 371-0014, Japan
}

Received February 12,2015; Accepted March 3, 2016

DOI: $10.3892 / \mathrm{ol} .2016 .4415$

\begin{abstract}
Cardiac metastasis of uterine cervical cancer with antemortem diagnosis is extremely rare. Therefore, its landscape epidemiology has not been well elucidated to date. In the present study, a case of solitary cardiac metastasis of uterine cervical cancer diagnosed antemortem is reported, and a review of the currently available literature (which includes 18 cases of cardiac metastasis of uterine cervical cancer) is conducted. In January 2013, a 78-year-old woman with squamous cell carcinoma (SCC) of the uterine cervix (International Federation of Gynecology and Obstetrics stage IIIb) underwent definitive radiotherapy at Gunma University Hospital (Gunma, Japan). Follow-up examination at 5 months after completion of the treatment indicated no evidence of recurrence or metastasis. In April 2014, the patient reported epigastric discomfort and general malaise. Electrocardiogram suggested myocardial dysfunction. Transthoracic echocardiography revealed the presence of a mass occupying the right ventricle and pericardial effusion. Cine magnetic resonance imaging demonstrated a filling defect in the right ventricle, and transcatheter biopsy confirmed SCC. The patient was diagnosed with a solitary cardiac metastasis of uterine cervical cancer. Despite aggressive medical therapy, the patient succumbed to disease 31 days after admission to hospital. A review of the current literature revealed that $84 \%$ of cases of cardiac metastasis develop within 2 years of completion of the initial treatment, and that electrocardiogram and echocardiography reveal findings of myocardial dysfunction and the presence of a mass in the right ventricle, respectively. A treatment strategy for cardiac metastasis of uterine cervical cancer has not been standardized thus
\end{abstract}

Correspondence to: Dr Takahiro Oike, Department of Radiation Oncology, Gunma University Graduate School of Medicine, 3-39-22 Showa-machi, Maebashi, Gunma 371-8511, Japan E-mail: oiketakahiro@gmail.com

Key words: uterine cervical cancer, cardiac metastasis, antemortem diagnosis, electrocardiogram, echocardiography far, and the prognosis is very poor, as the majority of patients succumbed to disease within 1 year. In summary, the current case and the literature review conducted in the present study suggest that: i) Cardiac metastasis should be included in the differential diagnosis in cases with nonspecific complaints such as epigastric discomfort and general malaise when patients have a history of uterine cervical cancer, particularly within the previous 2 years; and ii) electrocardiogram and echocardiography are convenient and effective modalities for the diagnosis of cardiac metastasis of uterine cervical cancer.

\section{Introduction}

Cardiac metastasis is relatively common in malignant neoplasms such as lung cancer, breast cancer, melanoma and lymphoma, with an incidence rate of $\sim 10-15 \%$ (1). In contrast to these types of malignancy, cardiac metastasis of uterine cervical cancer is rare (1-4). In autopsy cases of uterine cervical cancer, the incidence rate of cardiac metastasis has been reported to be $0.3-8.0 \%$ (1-4). Cardiac metastasis of uterine cervical cancer diagnosed prior to mortality (antemortem) is much rarer, as only $4.0 \%$ of cardiac metastasis cases are diagnosed antemortem (5). Among them, solitary metastasis to the heart is particularly rare, since cardiac metastasis is usually the result of the hematogenous spread of systemically disseminated disease (6). In the present study, a case of solitary cardiac metastasis of uterine cervical cancer diagnosed antemortem is reported, and a review of the literature is conducted.

\section{Case report}

A 78-year-old woman with squamous cell carcinoma (SCC) of the uterine cervix [Federation of Gynecology and Obstetrics stage IIIb (7)] underwent definitive radiotherapy in January 2013 at Gunma University Hospital (Gunma, Japan). The Eastern Cooperative Oncology Group/World Health Organization performance status (PS) (8) of the patient was 1. At the time of diagnosis, magnetic resonance imaging (MRI; Signa HDxt 1.5T; GE Healthcare Japan, Tokyo, Japan) revealed a bulky tumor $(66 \times 56 \times 45 \mathrm{~mm})$ at the uterine cervix with right parametrial invasion reaching the right pelvic wall. 
Computed tomography (CT; Aquilion ONE/ViSION Edition; Toshiba Medical Systems, Tochigi, Japan) and fluorine-18 fluorodeoxyglucose positron emission tomography (PET; Aquiduo PCA-7000B; Toshiba Medical Systems) indicated no evidence of metastasis, with the exception of an enlarged right obturator lymph node. Laboratory tests demonstrated elevated levels $(110.0 \mu \mathrm{g} / \mathrm{l}$; normal range, $<3.5 \mu \mathrm{g} / \mathrm{l})$ of serum SCC antigen. Radiotherapy (ONCOR Impression Plus; Siemens Japan, Tokyo, Japan) consisted of external beam radiotherapy and intracavitary brachytherapy. For external beam radiotherapy, whole pelvic irradiation of 50 Gy was delivered in 25 fractions over 5 weeks (the final 20 Gy dose was delivered using the central shielding technique), followed by boost irradiation to the right obturator lymph node of $6 \mathrm{~Gy}$ in 3 fractions at Gunma University Hospital. Weekly high-dose rate intracavitary brachytherapy using iridium-192 (Chiyoda Technol Corporation, Tokyo, Japan) delivered $24 \mathrm{~Gy}$ to point $\mathrm{A}(2 \mathrm{~cm}$ along the tandem from the external cervical os and $2 \mathrm{~cm}$ lateral to the tandem) in 4 fractions (over a duration of 7 weeks). Chemotherapy was not performed due to the advanced age of the patient. Five months after completion of the treatment, gynecologic examination of the uterine cervix and total body CT examination revealed no evidence of recurrence or metastasis. In addition, serum SCC antigen had decreased to levels of $0.8 \mu \mathrm{g} / 1$, which are within the normal limit.

In April 2014, the patient was admitted to our hospital with epigastric discomfort and general malaise, rapidly worsening over 3 days. The patient had a PS of 2 , in addition to high blood pressure $(152 / 115 \mathrm{mmHg}$; normal range, $<140 / 90 \mathrm{mmHg}$ ) and tachycardia (101 beats/min; normal range, 60-90 beats/min). Chest X-ray (RADSpeed Safire; Shimadzu Corporation, Kyoto, Japan) revealed enlargement of the heart (cardiothoracic ratio, $65 \%$; normal range, $<50 \%$ ). These findings suggested heart failure. Electrocardiogram (Vivid E9; Fukuda M-E Kogyo Co., Ltd., Tokyo, Japan) displayed low-voltage amplitudes in leads V1-V6 and T-wave inversion in leads V3-V6 (Fig. 1), suggesting myocardial dysfunction. Transthoracic echocardiography (Cardisuny 630; GE Healthcare Life Sciences) revealed the presence of a mass occupying the right ventricle and pericardial effusion, while cine MRI demonstrated a filling defect in the right ventricle (Fig. 2). These findings suggested the presence of a tumor in the right ventricle. A transcatheter biopsy from the right ventricle confirmed SCC. The levels of serum SCC antigen had elevated to $40.8 \mu \mathrm{g} / \mathrm{l}$. Total body CT examination indicated no evidence of local recurrence or metastasis to sites other than the heart. Taken together, these findings led to the diagnosis of solitary cardiac metastasis of uterine cervical cancer. Elevated levels of fibrin degradation products $(>20.0 \mu \mathrm{g} / \mathrm{ml}$; normal range, $0-4.0 \mu \mathrm{g} / \mathrm{ml}$ ), alongside thrombocytopenia $\left(1.1 \times 10^{4}\right.$ cells $/ \mu 1$; normal range, $16-35 \times 10^{4}$ cells $\left./ \mu 1\right)$ suggested disseminated intravascular coagulation (DIC) (9). Following admission, the hemodynamic status of the patient worsened gradually. Despite aggressive medical therapy, including intravenous administration of dobutamine (Shionogi, Inc., Osaka, Japan), noradrenaline (Daiichi Sankyo, Tokyo, Japan) and thrombomodulin alpha (Asahi Kasei, Tokyo, Japan), and transfusion of platelets and fresh frozen plasma
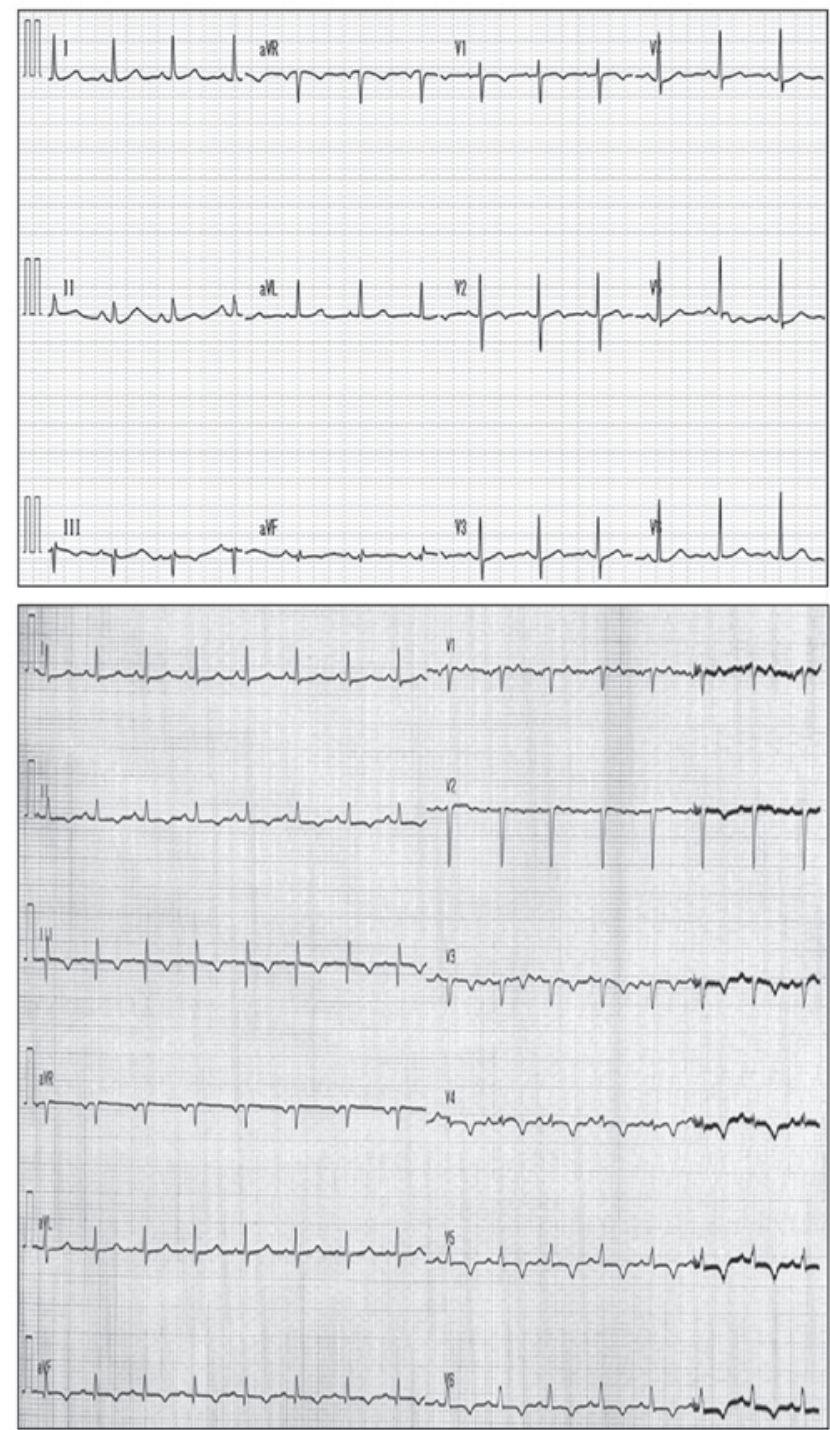

Figure 1. Electrocardiogram. Top panel, electrocardiogram at the time of initial diagnosis of uterine cervical cancer was unremarkable. Bottom panel, electrocardiogram at the time of diagnosis of cardiac metastasis revealed low-voltage amplitudes in V1-V6 and T-wave inversion in leads V3-V6.

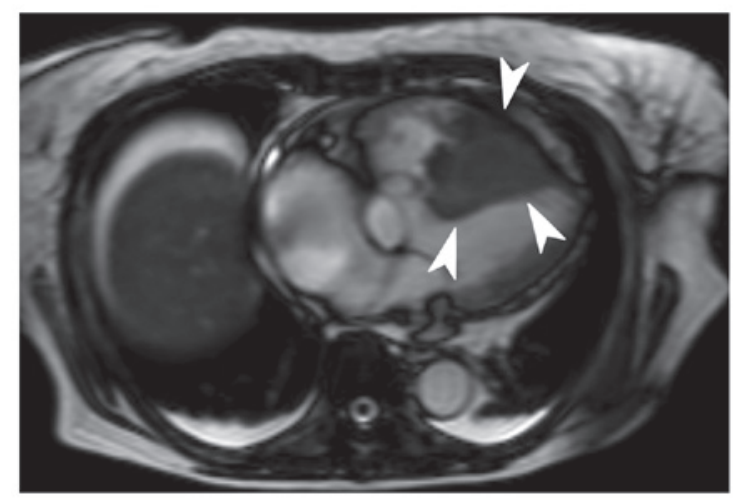

Figure 2. Cine magnetic resonance imaging revealed a filling defect in the right ventricle (as indicated by the white arrowheads).

(Japanese Red Cross Society, Tokyo, Japan) for a week, the patient succumbed to disease 31 days after admission to hospital. Permission for an autopsy was not granted at the 


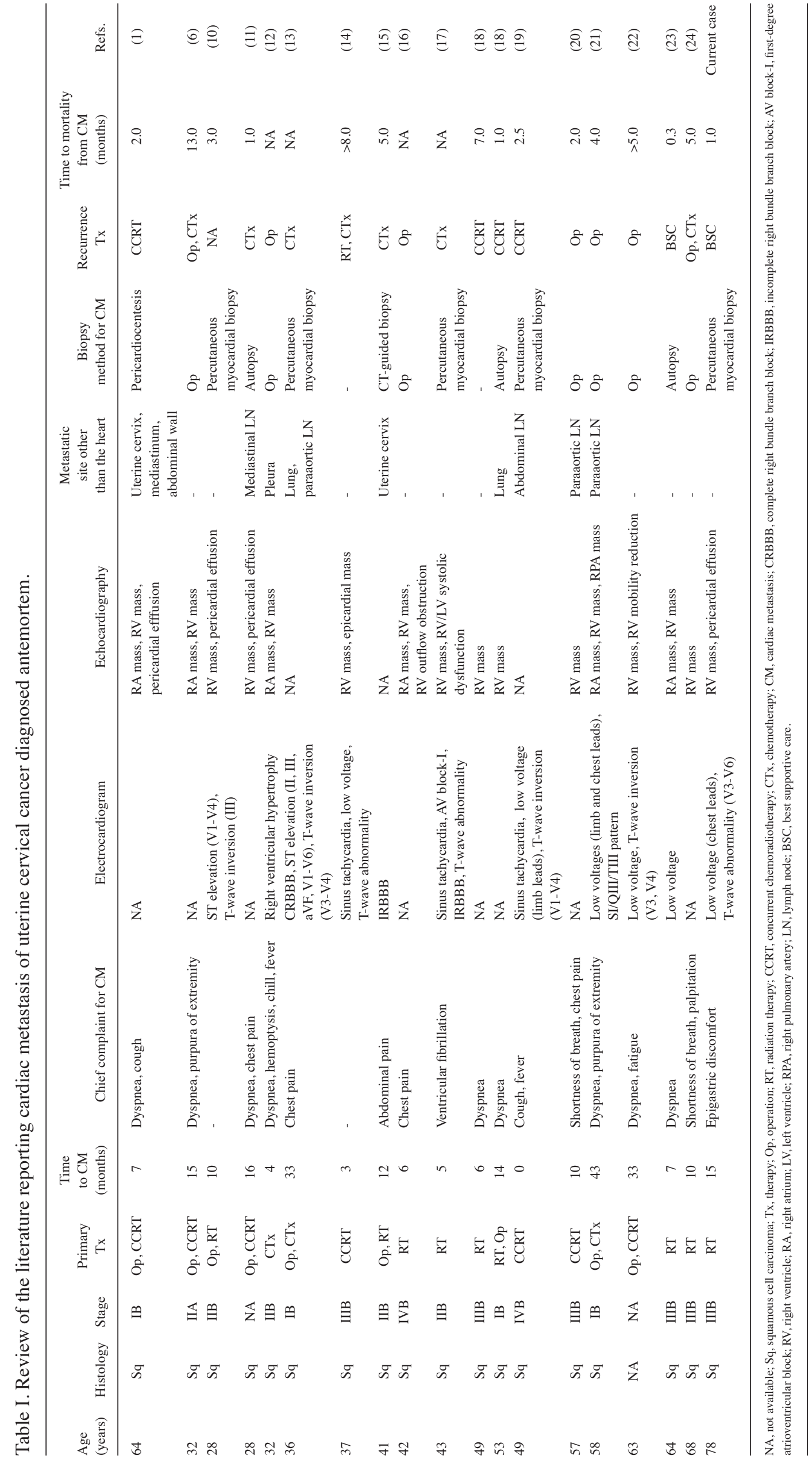



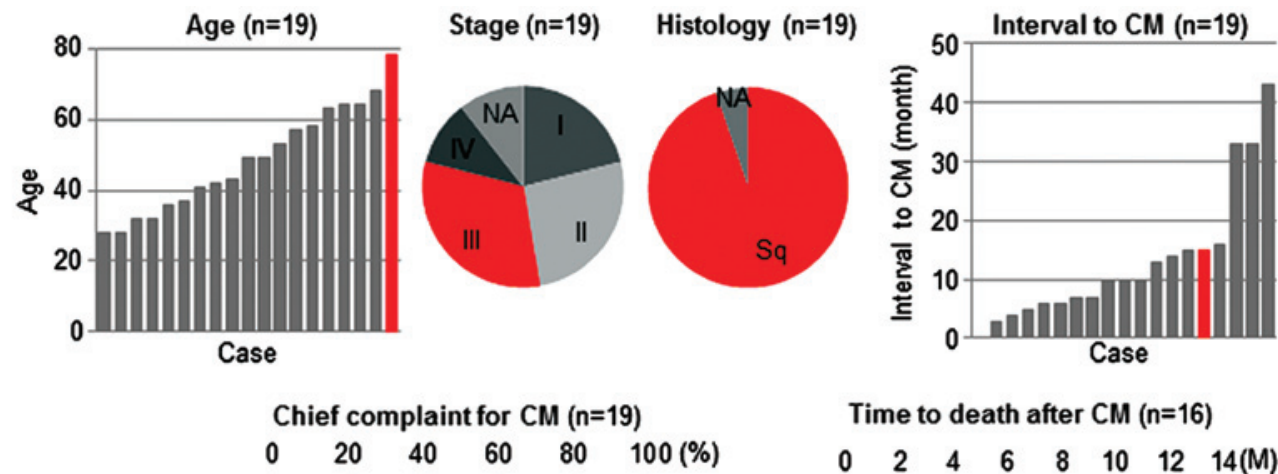

Time to death after CM ( $n=16)$
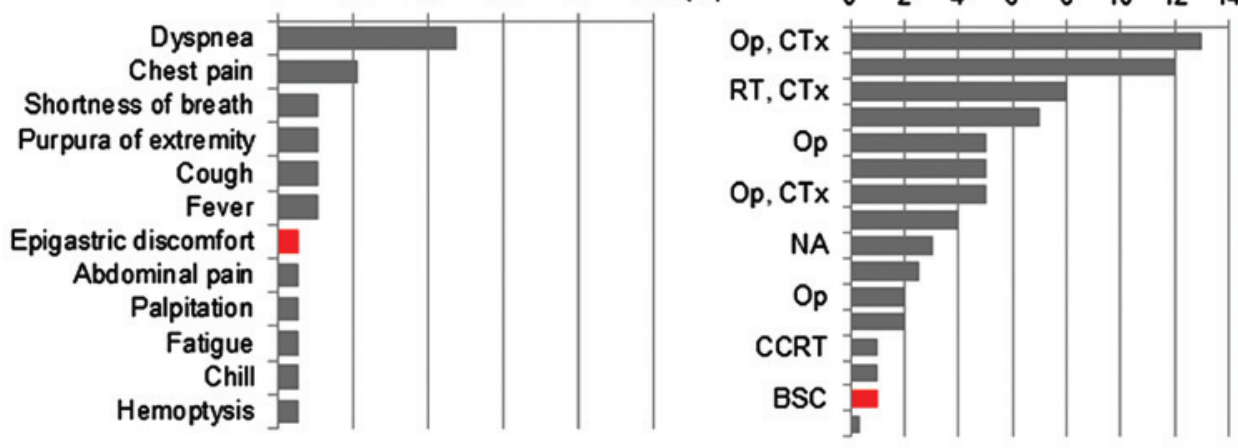

Electrocardiography $(n=10)$

$\begin{array}{lllllll}0 & 20 & 40 & 60 & 80 & 100 & (\%)\end{array}$

Echocardiography $(n=16)$

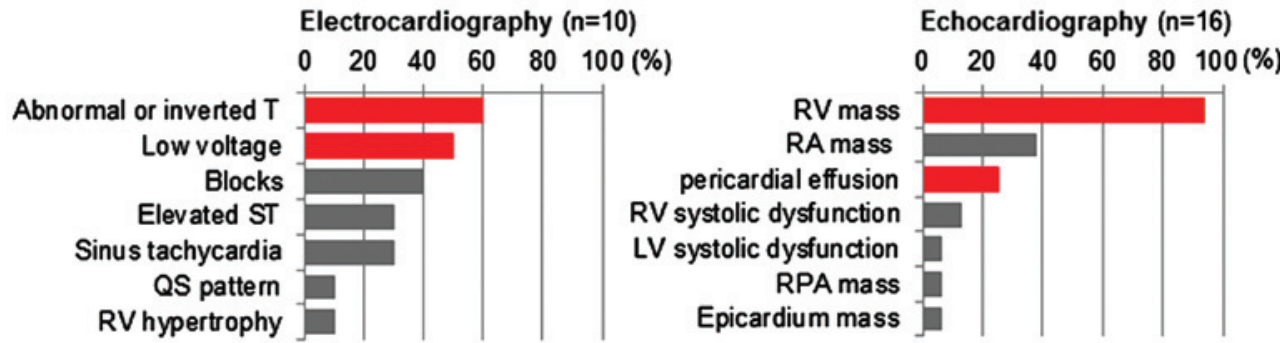

Figure 3. Graphical representation of the current and previously reported cases of cardiac metastasis of uterine cervical cancer diagnosed antemortem, indicating the patients' characteristics, examination findings, treatment modality and prognosis. Detailed information is described in Table I. The current case is indicated in red. NA, not available; Sq, squamous cell carcinoma; CM, cardiac metastasis; Op, operation; CTx, chemotherapy; RT, radiation therapy; CCRT, concurrent chemoradiotherapy; BSC, best supportive care; RV, right ventricle; RA, right atrium; LV, left ventricle; RPA, right pulmonary artery; M, months.

time of mortality, although the patient had provided written informed consent for publication of the present case.

\section{Discussion}

In the present study, a case of solitary cardiac metastasis of uterine cervical cancer diagnosed antemortem is reported. To the best of our knowledge, there have been only 18 cases of cardiac metastasis of uterine cervical cancer reported in the literature thus far (Table I, Fig. 3) (1,6,10-24). The number of cases showing solitary metastasis to the heart, as in the present case, is even smaller; with only nine cases reported to date $(6,10,14,16-18,22-24)$. The clinical stage at the time of initial diagnosis varied widely among the case reports published in the literature, ranging from stage I to IV (1,6,10-24). Cardiac metastasis occurred within 1 year after completion of the initial treatment in $63 \%$ of patients, and within 2 years in $84 \%$ of patients $(1,6,10-24)$. This indicates that the 2 years subsequent to the completion of the initial treatment is a critical period during which uterine cervical cancer patients should be carefully monitored for symptoms of cardiac metastasis. Dyspnea and chest pain were frequently reported as chief complaints in patients with cardiac metastasis $(1,6,11-13,16,18,20-23)$. Electrocardiogram frequently exhibited low voltages and T-wave abnormalities in chest leads, which is indicative of myocardial disability and/or pericardial effusion $(10,13,14,17,19,21-23)$. Echocardiography revealed a right ventricular mass in the majority of cases $(1,6,10-12,14,16-18,20-24)$. In the present case, it was not obvious to suspect cardiac metastasis from the complaints of epigastric discomfort and general malaise exhibited by the patient. Electrocardiogram revealed low-voltage amplitudes and T-wave inversion in chest leads, and echocardiograpy detected a mass in the right ventricle, which enabled to reach a diagnosis of cardiac metastasis. However, based on the current case and previous cases reported in the literature, no particular characteristics of solitary metastasis to the heart could be identified. Recent translational research indicates that molecules such as matrix metalloproteinase (MMP)-2 and MMP-9, and molecules associated with epithelial-mesenchymal transition such as Snail and vimentin, participate in cancer metastasis (25-28), which may be further elucidated via immunohistochemical analysis of these proteins. Taken together, the current case and the previous cases reported in the literature suggest that: i) Cardiac metastasis should be included in the differential diagnosis of patients with a history 
of uterine cervical cancer (particularly if diagnosed within the previous 2 years) who present with nonspecific complaints such as epigastric discomfort and general malaise; and ii) electrocardiogram and echocardiography are convenient and effective modalities for the diagnosis of cardiac metastasis.

The treatment strategy for cardiac metastasis of uterine cervical cancer has not been standardized to date, which is probably due to the extremely low incidence and the unfavorable prognosis of this disease (1). A review of the relevant literature suggests that the efficacy of aggressive treatment is controversial (1,6,10-24). Several reports suggest that aggressive treatment may prolong the patients' survival time $(6,18)$. For example, Byun et al (6) reported that a 32-year-old woman who underwent an open excision of an intracardiac tumor, followed by chemotherapy using carboplatin and paclitaxel, survived for 13 months after the diagnosis of cardiac metastasis. Lemus et al (18) reported that a 49-year-old woman who received $60 \mathrm{~Gy}$ of radiotherapy for an intracardiac tumor, combined with concurrent administration of 5-fluorouracil and cisplatin, survived for 7 months after the diagnosis of cardiac metastasis. By contrast, there are also reports indicating that aggressive treatment of cardiac metastasis does not always prolong the survival time of patients (Fig. 3) $(1,18,19)$. In several cases, intense concurrent chemoradiotherapy resulted in a survival time of only 3 months $(1,18,19)$. Thus, aggressive treatment to cardiac metastasis may not prolong the survival time when the patient develops systemic metastasis, although aggressive treatment could be beneficial if the cardiac metastasis is solitary $(1,6,18,19)$. Recent advances in diagnostic modalities, such as CT and PET, and their widespread use may increase the diagnostic yield of antemortem cardiac metastases, which may be controlled by aggressive treatment. In the present case, aggressive treatment such as surgery, radiotherapy or chemotherapy could not be performed due to the patient's unstable hemodynamic status and DIC. Therefore, the treatment strategy for cardiac metastasis of uterine cervical cancer should be carefully considered on a case-by-case basis.

In summary, the current case and the review of the literature conducted in the present study indicate that cardiac metastasis should be included in the differential diagnosis of patients with nonspecific complaints such as epigastric discomfort and general malaise, particularly if the patient has a previous history of uterine cervical cancer. In such cases, electrocardiogram and echocardiography may be convenient and effective modalities for the diagnosis of cardiac metastasis.

\section{Acknowledgements}

The present study was supported by the Strategic Young Researcher Overseas Visits Program for Accelerating Brain Circulation of the Japan Society for the Promotion of Science (Tokyo, Japan).

\section{References}

1. Kim HS, Park NH and Kang SB: Rare metastases of recurrent cervical cancer to the pericardium and abdominal muscle. Arch Gynecol Obstet 278: 479-482, 2008.

2. Badib AO, Kurohara SS, Webster JH and Pickren JW: Metastasis to organs in carcinoma of the uterine cervix. Influence of treatment on incidence and distribution. Cancer 21: 434-439, 1968.
3. Peeples WJ, Inalsingh CH, Hazra TA and Graft D: The occurrence of metastasis outside the abdomen and retroperitoneal space in invasive carcinoma of the cervix. Gynecol Oncol 4: 307-310, 1976

4. Klatt EC and Heitz DR: Cardiac metastases. Cancer 65: 1456-1459, 1990.

5. Greenwald EF, Breen JL and Gregori CA: Cardiac metastases associated with gynecologic malignancies. Gynecol Oncol 10: 75-83, 1980.

6. Byun SW, Park ST, Ki EY, Song H, Hong SH and Park JS: Intracardiac metastasis from known cervical cancer: A case report and literature review. World J Surg Oncol 11: 107, 2013.

7. Pecorelli S. Revised FIGO staging for carcinoma of the vulva, cervix, and endometrium. Int J Gynaecol Obstet 105: 103-104. 2009.

8. Oken MM, Creech RH, Tormey DC, et al: Toxicity and response criteria of the Eastern Cooperative Oncology Group. Am J Clin Oncol 5: 649-655, 1982.

9. Wada H, Gabazza EC, Asakura H, et al: Comparison of diagnostic criteria for disseminated intravascular coagulation (DIC): Diagnostic criteria of the International Society of Thrombosis and Hemostasis and of the Japanese Ministry of Health and Welfare for overt DIC. Am J Hematol 74: 17-22, 2003.

10. Kountz DS: Isolated cardiac metastasis from cervical carcinoma: Presentation as acute anteroseptal myocardial infarction. South Med J 86: 228-230, 1993.

11. Senzaki H, Uemura Y, Yamamoto D, et al: Right intraventricular metastasis of squamous cell carcinoma of the uterine cervix: An autopsy case and literature review. Pathol Int 49: 447-452, 1999.

12. Krivokapich J, Warren SE, Child JS, et al: M-mode and cross-sectional echocardiographic diagnosis of right ventricular cavity masses. J Clin Ultrasound 9: 5-10, 1981.

13. ShimotsuY,IshidaY,FukuchiK,etal:Fluorine-18-fluorodeoxyglucose PET identification of cardiac metastasis arising from uterine cervical carcinoma. J Nucl Med 39: 2084-2087, 1998.

14. Feys A, Herregods MC and Ector H: Cardiac metastasis from a stage IIIb cervix carcinoma. Acta Cardiol 60: 73-75, 2005.

15. Ando K, Kashihara K, Harada M, Kasem I, Nishitani H, Sano N and Ohtani T: Carcinoma of the uterine cervix with myocardial metastasis. Gynecol Oncol 65: 169-172, 1997.

16. Borsaru AD, Lau KK and Solin P: Cardiac metastasis: A cause of recurrent pulmonary emboli. Br J Radiol 80: e50-e53, 2007.

17. Batchelor WB, Butany J, Liu P and Silver MD: Cardiac metastasis from primary cervical squamous cell carcinoma: Three case reports and a review of the literature. Can J Cardiol 13: 767-770, 1997.

18. Lemus JF, Abdulhay G, Sobolewski C and Risch VR: Cardiac metastasis from carcinoma of the cervix: Report of two cases. Gynecol Oncol 69: 264-268, 1998.

19. Iwaki T, Kanaya H, Namura M, et al: Right ventricular metastasis from a primary cervical carcinoma. Jpn Circ J 65: 761-763, 2001.

20. Nakao Y, Yokoyama M, Yasunaga M, et al: Metastatic tumor extending through the inferior vena cava into the right atrium: A case report of carcinoma of the uterine cervix with para-aortic lymph node metastases. Int J Gynecol Cancer 16: 914-916, 2006.

21. Inamura K, Hayashida A, Kaji Y, et al: Recurrence of cervical carcinoma manifesting as cardiac metastasis three years after curative resection. Am J Med Sci 328: 167-169, 2004.

22. Ferraz JG, Martins AL, de Souza JF, Matos A, Canto AP and Martins AM: Metastatic tumor of squamous cell carcinoma from uterine cervix to heart: Ante-mortem diagnosis. Arq Bras Cardiol 87: e104-e107, 2006 (In English, Portuguese).

23. Mohammed S and Khodadoust K: Carcinoma of the cervix causing massive intracardiac embolus. Gynecol Oncol 56: 294-297, 1995.

24. Saitoh Y, Aota M, Koike H, Nakane T, Iwasa Y and Konishi Y: Isolated right ventricular metastasis of uterine cervical carcinoma. Jpn J Thorac Cardiovasc Surg 53: 645-648, 2005.

25. Wang C, Ma HX, Jin MS, et al: Association of matrix metalloproteinase (MMP)-2 and -9 expression with extra-gastrointestinal stromal tumor metastasis. Asian Pac J Cancer Prev 15: 4187-4192, 2014.

26. Chen L, Zhou Q, Xu B, Liu J, Shi L, Zhu D, Wu C and Jiang J: MT2-MMP expression associates with tumor progression and angiogenesis in human lung cancer. Int J Clin Exp Pathol 7: 3469-3477, 2014.

27. Lee MY and Shen MR: Epithelial-mesenchymal transition in cervical carcinoma. Am J Transl Res 4: 1-13, 2012

28. Chen Z, Li S, Huang K, Zhang Q, Wang J, Li X, Hu T, Wang S, Yang R, Jia Y, et al: The nuclear protein expression levels of SNAI1 and ZEB1 are involved in the progression and lymph node metastasis of cervical cancer via the epithelial-mesenchymal transition pathway. Hum Pathol 44: 2097-2105, 2013. 